\title{
NEGATIVE FREQUENCY DEPENDENCE AND THE IMPORTANCE OF SPATIAL SCALE
}

\author{
Jane Molofsky, ${ }^{1,5}$ James D. Bever, ${ }^{2}$ Janis Antonovics, ${ }^{3}$ And Timothy J. Newman ${ }^{4}$ \\ ${ }^{1}$ Department of Botany, University of Vermont, Burlington, Vermont 05405 USA \\ ${ }^{2}$ Department of Biology, Indiana University, Bloomington, Indiana 47405 USA \\ ${ }^{3}$ Biology Department, University of Virginia, Charlottesville, Virginia 22903-2477 USA \\ ${ }^{4}$ Department of Physics, University of Virginia, Charlottesville, Virginia 22903-2477 USA
}

Abstract. Issues of spatial scale are inherent in many ecological systems. This study uses a spatially explicit cellular automaton model to explore how the scale of dispersal interacts with the scale and strength of negative frequency dependence to determine patterns of species distribution. Counter to expectation, strong local frequency-dependent interactions result in random spatial patterns. When dispersal scale and interaction scale are decoupled, the resulting patterns are not necessarily random. For strong negative frequency dependence, stable bands result when the scale of interaction exceeds the scale of dispersal, and bands with two-point cycles result when the scale of dispersal exceeds the scale of interaction. However, for weaker interactions occurring over intermediate scales, only random patterns result. Thus, our results call into question the utility of inferring any ecological interaction from only the spatial distributions of the putatively interacting species. Furthermore, our results call for new experimental studies that explicitly manipulate the strength and the scale of the processes being studied.

Key words: cellular automata; competition scale; dispersal scale; negative frequency dependence and pattern; pattern vs. process; population dynamics, scale and process; spatial patterns; spatial scale; stochastic spatial model.

\section{INTRODUCTION}

Investigation and documentation of patterns to gain insight into process has had a long history in ecology (Pielou 1977, Greig-Smith 1983, Kershaw and Looney 1985). Much of the debate has centered around whether one can infer the occurrence of competition based on the presence or absence of particular species combinations in adjacent communities (e.g., Connor and Simberloff 1984, Gilpin and Diamond 1984, Gilpin et al. 1984). In this context, random patterns are assumed to imply that no strong interactions have taken place. To distinguish patterns generated from competition (or other potential mechanisms), from patterns generated without competition, proponents of null models have proposed re-randomization tests (Gotelli and Graves 1996). However, strong competitive interactions can give rise to random species associations (Rummel and Roughgarden 1983, Cale et al. 1989). Moreover, multiple processes may contribute to the generation of community patterns (Cale et al. 1989). Further complicating our understanding of how processes influence patterns are issues of spatial scaling (e.g., Levin 1992). Each eco-

Manuscript received 30 May 2000; revised 26 April 2001; accepted 7 May 2001.

${ }^{5}$ E-mail: jmolofsk@zoo.uvm.edu logical process may operate over a unique spatial scale, and it may be necessary to know this scale in order to adequately predict the final dynamics (Levin 1992).

One important process that can influence the generation of patterns in communities is the scale of dispersal (Greig-Smith 1983, Pacala and Silander 1985). For example, local dispersal can cause deviations from random patterns even when competition between species is absent (Greig-Smith 1983, Pacala and Silander 1985, Pacala 1987). This is also a well-known result in population genetics (Wright 1943, Turner et al. 1982). Reaction diffusion models that include both competition and dispersal can result in stable species aggregations (Murray 1989). In spite of these theoretical results the importance of dispersal in altering patterns predicted from competition models is not widely appreciated (Gotelli and Graves 1996).

Interactions between species and between genotypes often result in frequency-dependent dynamics. For example, niche partitioning will result in negative frequency dependence within systems of competition (Ayala 1971, Antonovics and Kareiva 1988). Predation (Clarke 1969), parasitism (May and Anderson 1983), and mutualism (Bever 1999) can also result in negative frequency dependence. This negative frequency dependence has been the center of great interest within 
ecology and evolution because of its potential importance in maintaining diversity. A number of experimental field studies have clearly shown the importance of frequency-dependent selection in nature (Antonovics and Ellstrand 1984, Kelley et al. 1988). In a previous study, Molofsky et al. (1999) showed that strong negative frequency dependence could generate a random spatial pattern so that a field ecologist might mistakenly conclude that no strong species interactions were present. In the present paper, we extend the previous study to address how negative frequency dependence and dispersal operate over different scales to affect spatial patterning in communities.

\section{Model Development}

The model was developed for two species competing on a two-dimensional grid. Each location on the grid contains either one of two different species or is empty. Thus, each position on the grid can be visualized as a specific location in space. The details of how individuals are positioned on the grid depend upon the initial starting conditions chosen. The dynamics develop from the application of transition rules in concert over the entire grid. For most of the initial conditions studied, all individuals die simultaneously at the end of each generation so that each site is recolonized each generation, as might be appropriate for annual plants. However, for some initial conditions, we relaxed this assumption and instead updated each cell in the grid at random, as might be more appropriate for organisms with overlapping generations.

The transition rules involve the interaction of two processes, interspecific interaction and dispersal. For a given cell, each of the processes depends upon the proportion of each species in the surrounding cells over some finite distance-hence, each process is frequency dependent. However, the outcome of interspecific interactions can be negatively dependent upon the proportion of each species in its surrounding neighborhood, while the outcome of dispersal is always strictly proportional to the number of each species in the neighborhood. Dispersal within the dispersal neighborhood is uniform but drops to zero outside of the dispersal neighborhood. The "interaction neighborhood" refers to the neighbors that are used to determine the negative frequency-dependence probability; and the "dispersal neighborhood" refers to the neighbors that are used to determine the dispersal probability. In order to investigate the effect of the changing scale of interspecific interactions and dispersal, we constructed general rules that are scale neutral. The size of these two neighborhoods can vary from one square to the size of the entire grid. At this large neighborhood, the model reduces to a stochastic model without spatial structure.

The probability of a site being occupied by a particular species, such as species 1 , is given by the following equation:

$$
P_{1}=H_{1} \times D_{1} /\left(H_{1} \times D_{1}+H_{2} \times D_{2}\right) .
$$

$H_{1}$ and $H_{2}$ represent the habitat quality resulting from frequency dependence for species 1 and 2, respectively, in the interaction neighborhood. $D_{1}$ and $D_{2}$ represent the number of individuals of the two species that dispersed into the target cell from the dispersal neighborhood. We assume that at the end of each generation individuals produce a fixed number of offspring that are dispersed uniformly over the entire dispersal neighborhood. The spatial scale of habitat quality and dispersal is completely adjustable. To make the transition rules stochastic, we determined site occupancy by selecting a uniformly distributed random number, $x$, and if $P_{1} \leq x$, then the site is occupied by species 1 , otherwise it is occupied by species 2 .

The habitat quality controls the interspecific interaction neighborhood and is determined by the following equation:

$$
H_{1}=0.5+a\left(f_{1}-0.5\right) .
$$

Habitat quality of the cell is a measure of the relative likelihood of establishment of a single propagule of species 1 relative to a single propagule of species 2 . The parameter, $f_{1}$, is the proportion of species 1 in the adjacent cells over some predefined neighborhood scale. The spatial scale of interspecific interaction neighborhood can be varied by adjusting the range of neighboring cells used to calculate the frequency of each type in the neighborhood. The parameter $a$ represents the strength of frequency dependence and can vary between -1 and 1 . If $a=0$, then $H_{1}=0.5$ and neither positive nor negative frequency dependence occurs. In this case, each species establishes in proportion to their relative frequency in the dispersal neighborhood. This is the neutral case, which is equivalent to the voter model (Holley and Liggett 1975), and is also equivalent to a model of random drift (Wright 1943). Negative frequency dependence occurs when $a<0$.

The sizes of the interaction and dispersal neighborhoods were varied from neighborhoods consisting of 9 squares to 1849 squares. To change the size of neighborhoods incrementally, we used square neighborhoods of size $=(2 y-1)^{2}$, where $y$ increases incrementally from 2 up to 22 . For example, for $y=2$, the neighborhood size is 9 cells (the center square and all eight squares that it touches) and when $y=6$, the neighborhood size is 121 cells.

Although the model greatly simplifies the ecological interactions that occur between species, it is still not possible to obtain analytical solutions that take into account the explicit spatial structure. Therefore, we used a computer simulation of a spatially explicit stochastic model in which the probability of species 1 establishing in a given cell is determined by the composition of the neighboring cells. To ensure that our results were not dependent on the details of how we constructed the simulation, we explored how differ- 
ences in the simulation structure affected the outcome. Changes that we investigated included (1) square and circular neighborhoods, (2) different initial frequencies of the two species (10:90, 20:80, 50:50), (3) clumped and random initial arrangements of species on the grid, (4) torus, reflective, and absorbing boundary conditions, and (5) grid sizes varying from $100 \times 100$ to $500 \times 500$. Results of the simulations were not altered by any of these changes. In simulations where the placement of each species on the grid was chosen at random, we chose 20 different initial arrangements of individuals. Differences in the initial configurations of individuals did not alter the results.

In addition, we also investigated a variety of different ecological situations such as the presence of demographic stochasticity, the presence of unsuitable sites within the habitat, and asymmetrical negative frequency dependence. To simulate demographic stochasticity, we randomly removed individuals from the grid each generation. To calculate the neighborhood frequency in the presence of these removals, we used the probability $(d)$ that a given site will be unoccupied in each generation. The probability of establishment of the two species was then adjusted by this probability as follows:

$$
P_{1}^{\prime}=(1-d) P_{1} \text {, and } P_{2}^{\prime}=(1-d)\left(1-P_{1}\right)
$$

where $P_{1}^{\prime}, P_{2}^{\prime}$ are the adjusted probability of establishment of species 1 and species 2 , respectively. The results of our simulations were unchanged for levels of demographic stochasticity ranging from $10 \%$ to $50 \%$ of the entire grid. We also considered situations that would simulate environmental heterogeneity resulting in some sites being unsuitable for colonization. Here, we considered a set proportion of the grid unavailable for colonization at the start of the simulations. We created unsuitable areas of different size in the following manner. Prior to the start of the simulation, the grid was initialized with a certain proportion of sites that could not be colonized. For unsuitable areas larger than a single grid cell, we increased the probability that sites next to a site that was already chosen as unsuitable would also be unsuitable. By varying this probability, we could increase the size of the unsuitable area in the grid, while holding the amount of area that was unavailable for colonization constant. Changing the amount and spatial autocorrelation of sites available for colonization did not change the qualitative results. Finally, we simulated conditions where the species differed in the degree to which they experienced negative frequency dependence. Having asymmetrical frequency dependence did not change the qualitative results. However, the species that experienced less negative frequency dependence had a lower final equilibrium density than the species that had higher negative frequency dependence.

Each simulation was run until the resulting pattern had stabilized. The steady state of the model is not a particular configuration, but rather a particular form of the underlying probability distribution. The time for the final pattern to emerge depends upon the grid size and relative scales of interaction and dispersal. Three types of pattern were observed: random, clustered, or sharply banded. To distinguish between spatial patterns, at the end of each simulation, we calculated a clustering index (CI). The clustering index (CI) is the average value of the difference between the observed frequency of neighbors in the interaction neighborhood and the expected frequency of neighbors if species were distributed at random on the grid corrected for by the final global frequency represented in the grid. We calculated the $\mathrm{CI}$ index for model conditions that are known to result in a random pattern (i.e., $a=-1$, local scales, see Results: Equal scales of ..., below) for 1000 different initial conditions. For the known random pattern, the CI was very close to zero $(\mathrm{CI}=0.0018,1$ $\mathrm{SD}=0.0026, N=1000)$. For each of the other conditions, 20 simulations were run and the mean and standard deviation of CI are reported.

\section{REsults}

The results described below, unless otherwise noted, hold for square and circular neighborhoods, discrete and overlapping generations, all grid sizes, all boundary conditions, random and clumped initial spatial arrangements, the presence of demographic stochasticity ranging from 10 to $50 \%$ of the entire grid, and the presence of unsuitable sites ranging from 10 to $50 \%$ of the entire grid that are either distributed at random or spatially autocorrelated. Unless otherwise indicated all specific results reported below are for discrete generations, random initial spatial arrangements of individuals, no demographic stochasticity, uniform habitat (i.e., no unsuitable habitat) and grid sizes of $100 \times$ 100 with absorbing boundaries. The local spatial scale equals the 9-cell neighborhood and the large spatial scale equals the 1849-cell neighborhood.

\section{Equal scales of negative frequency dependence and dispersal}

At local spatial scales (interaction and dispersal neighborhood size of nine cells), weak negative frequency dependence results in clumped spatial patterns, which are larger when the interactions are weaker $(a$ $=-0.01, \mathrm{CI}$ [clustering index] $=0.23,1 \mathrm{SD}=0.04$, $N=20$ replicates; $a=-0.1, \mathrm{CI}=0.09, \mathrm{SD}=0.004$, $N=20$; Fig. 1). However, strong negative frequency dependence $(a=-1)$ at local spatial scales leads to random patterns $(\mathrm{CI}=0.0005, \mathrm{SD}=0.003, N=20$; Fig. 1). This can be shown analytically. With strong negative frequency dependence $(a=-1)$, then $H_{1}$ (habitat quality for species 1 ) $=1-f_{1}$ and $D_{1}$ (the number of individuals of species 1 that dispersed into the target cell from the dispersal neighborhood) $=f_{1}$ (where the parameter $f_{1}$ is the proportion of species 1 in the adjacent cells over some predefined neighborhood scale)

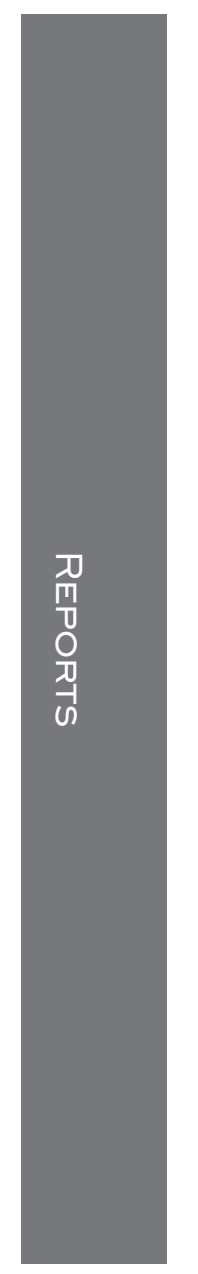




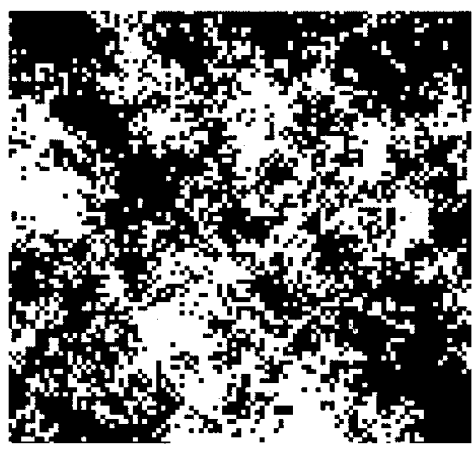

Weak frequency dependence $a=-0.01$

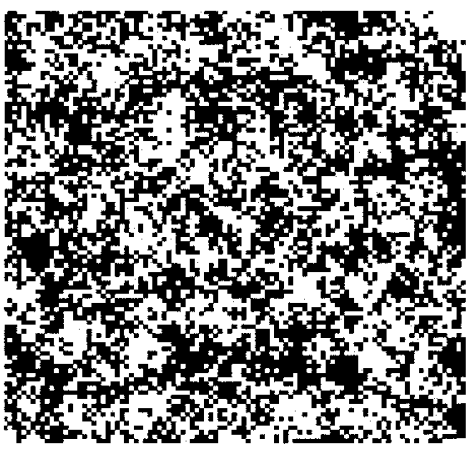

Weak frequency dependence $a=-0.1$

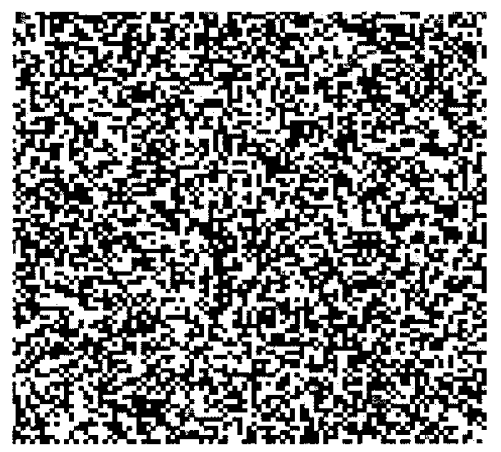

Strong frequency dependence $a=-1$

FIG. 1. Patterns that develop after 1000 generations for one initial distribution for the nine-cell neighborhood subject to different levels of negative frequency dependence ranging from very weak to strong. Simulations were initialized with equal frequencies of each species randomly positioned on the grid. All simulations were run with discrete generations, $100 \times 100$ grid size, and absorbing boundary conditions.

and the probability of a cell being occupied by species 1 becomes

$$
\begin{aligned}
P_{1}= & {\left[\left(1-f_{1}\right) \times f_{1}\right] /\left\{\left[\left(1-f_{1}\right) \times f_{1}\right]\right.} \\
+ & {\left.\left[f_{1} \times\left(1-f_{1}\right)\right]\right\}=0.5 . }
\end{aligned}
$$

This indicates that the transition probability for each species is random.

When the spatial scale of both negative frequencydependent interaction and dispersal are increased simultaneously (interaction and dispersal neighborhood of 1849 cells), the clumped patterns found for weak negative frequency-dependent interactions disappear and only random patterns are produced.

\section{Scale of negative frequency dependence less than that of dispersal}

When negative frequency-dependent interactions are weak $(a=-0.1)$, random patterns develop $(\mathrm{CI}=0.002$, $\mathrm{SD}=0.002, N=20$; Fig. $2 \mathrm{~A})$. The random spatial pattern results because dispersal reintroduces rare types into a location but as the frequency of rare types increases, the negative frequency dependence is too weak to cause a complete shift from the formerly rare but now common types to the new rare types.

Under strong negative frequency dependence ( $a=$ $-1)$, bands develop when the scale of interaction is local and dispersal scale is large $(\mathrm{CI}=0.369, \mathrm{SD}=$ $0.005, N=20$; Fig. 2B). These bands are not stable but represent a global two-point cycle. The bands develop because one type is initially more common in a local region than the other type, making the rare type more fit in that location. However, because of high dispersal, the rare type disperses into the region of the common type and increases in abundance. As dispersal makes the previously rare type the common type, the fitness of the two types reverses and band locations of the two types alternate.
Scale of negative frequency dependence greater than that of dispersal

When dispersal occurs locally and the interaction scale is larger, two spatial patterns (clumps or bands) can result. Which pattern develops depends upon the strength of the negative frequency-dependent interactions.

If the negative frequency-dependent interactions are weak $(a=-0.1)$, clumps of individuals of each species form $(\mathrm{CI}=0.18, \mathrm{SD}=0.011, N=20$; Fig. 2C). If frequency dependence is weak, there is a scale/distance beyond the clump at which a neighborhood can be found where the alternative type is in the majority. This then serves to reinforce the clumping, as the broad interaction effect ensures that the cells in the clumped region have a frequency-dependent minority advantage (being in the minority over this scale); in addition, local dispersal continues to reinforce the clumping. The weak interactions prevent the clumps from forming into sharp bands.

If the negative frequency dependence is strong ( $a=$ $-1)$, then stable and persistent bands develop throughout the landscape $(\mathrm{CI}=0.372, \mathrm{SD}=0.0066, N=20$; Fig. 2D). In the case of both weak and strong frequency dependence, the initial clumping that precedes pattern formation is undoubtedly due to stochastic forces enhanced by the local dispersal. Clumps form initially because dispersal is very local, and overall frequencies in the neighborhoods are equal. When frequency dependence is very strong, aggregates form and are reinforced and molded into sharp bands of equal width by negative frequency dependence. Negative frequency dependence reinforces the bands because the neighboring bands of opposite type are within the interaction neighborhood, thereby making the central band type the minority at that large scale. 


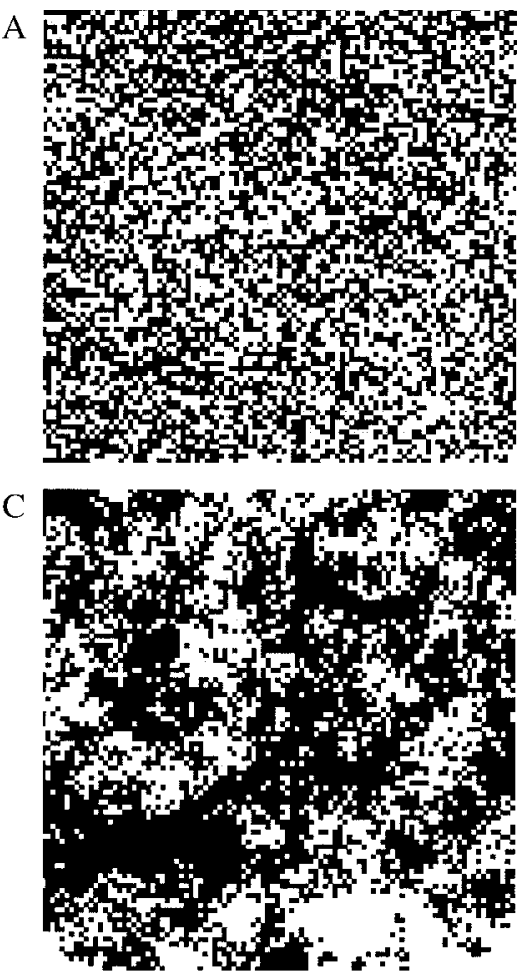

Weak frequency dependence $a=-0.01$
B

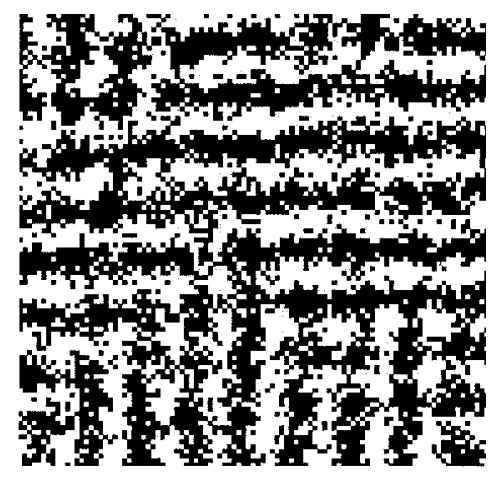

D

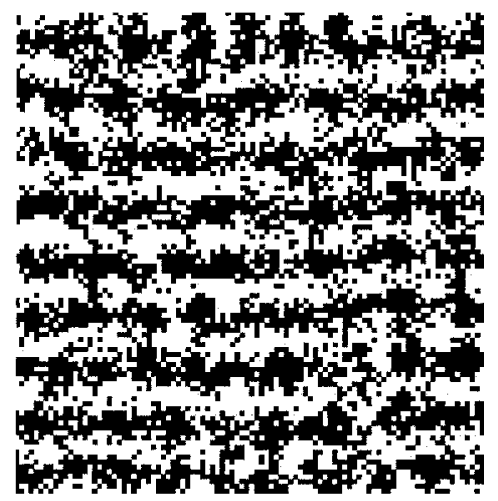

Strong frequency dependence $a=-1$

FIG. 2. Patterns that develop after 1000 generations for one initial distribution for the cases where scales are unequal for two different strengths of frequency-dependent interactions. In the top panels, dispersal is greater than frequency-dependent interaction (interaction scale $=9$ cells; dispersal $=1849$ cells) for $(\mathrm{A})$ weak frequency dependence $(a=-0.01)$ and $(\mathrm{B})$ strong frequency dependence $(a=-1)$. In the bottom panels, frequency-dependent interaction is greater than dispersal (interaction scale $=1849$ cells; dispersal $=9$ cells $)$ for $(C)$ weak frequency dependence $(a=-0.01)$ and (D) strong frequency dependence $(a=-1)$. Simulations were initialized with equal frequencies of each species randomly positioned on the grid. All simulations were run with discrete generations, $100 \times 100$ grid size, and absorbing boundary conditions.

\section{DISCUSSION}

Patterns in species associations have been used by ecologists to infer mechanisms for many years (Pielou 1977, Greig-Smith 1983, Kershaw and Looney 1985). However, similar spatial patterns may result from multiple mechanisms. Using a simple simulation model that explicitly included resource-based competition, Rummel and Roughgarden (1983) and Cale et al. (1989) demonstrated that strong competitive interactions between species can generate random associations. We found that random patterns could also result from negative frequency dependence, either weak or strong, provided that the interaction and dispersal occurred over the same spatial scale. Clearly, random patterns are not adequate null hypotheses for the absence of interactions (Gotelli and Graves 1996). Our results further highlight the difficulties involved in inferring process from pattern because we found that (1) the pattern generated by a given process depends critically on the relative scale over which interaction and dispersal occur, and (2) particular individual patterns can be generated by very different mechanisms.
The patterns generated by negative frequency dependence depended strongly on the relative spatial scale over which dispersal and interaction occurred. When these processes occurred at very different spatial scales, clumped and banded patterns were generated. When the scale of dispersal was small relative to the scale of interaction, negative frequency dependence generated a clumped and even sharply banded pattern. In this case, the bands generated by strong negative frequency dependence were stable over time. Clumps and bands can also be generated by negative frequency dependence when the scale of dispersal is large relative to the scale of interaction. The locations of the bands generated by this circumstance, however, are not static but show a two-point cycle. Our results were robust under a wide range of starting conditions, including clumped and random initial arrangements of species, different initial starting frequencies of species, in the presence of demographic stochasticity, and in landscapes containing unsuitable habitat. Moreover, clumping and band development were not critically dependent on the shape of the interaction areas: both ar- 
rangements occurred in square and circular interaction areas. Because our cellular automaton model was constructed with simultaneous (or parallel) updating across the whole region, it was designed to reflect discrete generations as might be seen with an annual plant. However, we also investigated a range of parameter values where there was random updating. It is therefore particularly interesting that the formation of stable bands occurs with random updating as well as with parallel updating. However, the phenomenon of a twopoint cycle of groups or bands of cells exchanging types disappears completely with random updating. The patterns remain the same, but the dynamics are very different.

Likewise, similar patterns can be generated by very different processes, including opposite processes of positive and negative frequency dependence (Molofsky et al. 2001). For example, clumped patterns can be generated by weak negative frequency dependence, random drift, or positive frequency dependence. However, the ultimate dynamical outcome under these processes can be quite different, with two types being deterministically maintained under negative frequency dependence, while one of the two types may be lost under drift or positive frequency dependence (Molofsky et al. 2001). Moreover, strongly banded patterns can be generated and maintained by either strong negative frequency dependence or strong positive frequency dependence (Molofsky et al. 2001). Clearly, it would be difficult to distinguish the clumped or banded patterns produced through negative frequency dependence from those produced through neutral or positive frequency dependence based on pattern data alone.

Sharp and stable boundaries between community types are a common feature in many landscapes (GreigSmith and Chadwick 1965, White 1971, Wilson and Agnew 1992). Their occurrence has been attributed to either sharp changes in the environment (Gleason 1939) or positive feedback mechanisms created by the species themselves (Wilson and Agnew 1992, Molofsky et al. 2001). Differences in dispersal and interaction scales is an equally plausible mechanism for creating these sharp boundaries.

Our results have implications for the dynamics of many ecological situations. Negative frequency dependence has been documented in many different ecological situations such as competition (Ayala 1971), pollination (Smithson and McNair 1996), predation (Clarke 1969), and parasitism (May and Anderson 1983). However, we do not know how the strength of frequency dependence measured in field studies compares to the values we use in this model. Nevertheless, even if precise measures of frequency dependence could be determined, until more is known about the scale over which the frequency-dependent process occurs, it is impossible to determine what kind of pattern should be predicted. There are numerous biological examples of ecological processes that operate over dif- ferent spatial scales. For example, in plant populations, the distance over which seeds disperse and pollinators transfer pollen (e.g., Levin and Kerster 1974, Price and Waser 1979) can be large, while interactions that generate frequency dependence, including competition (Pacala and Silander 1985, Silander and Pacala 1985, Molofsky 1999) and feedback through the soil community (Bever 1994, Bever et al. 1997), can occur over small distances. The reverse scenario (i.e., local dispersal but long-range negative interactions) is also possible. Negative frequency dependence over large spatial scales may be generated by a wide-ranging predator. For example, in grassland ecosystems, many perennial-grass species reproduce clonally and thus disperse locally, while large herbivores such as ungulates may forage over large areas (Frank et al. 1998). If these herbivores selectively feed on the more common species (Augustine and McNaughton 1998), then negative frequency dependence will result.

Determining the relative interaction strengths between species is critical for determining which species will persist in communities (Paine 1980, Wooton, 1994, Berlow et al. 1999). Additionally, issues of spatial scale are inherent in many ecological systems and the importance of determining the correct scale for theoretical or empirical studies has been emphasized before (Kareiva and Anderson 1988, Levin 1992). Several theoretical studies have addressed the mathematical procedures for determining the most appropriate scale of study for a particular ecological community (Levin 1992, Pascual and Levin 1999). In addition, theoretical studies have addressed whether spatially explicit models provide different answers to important ecological questions than do non-spatial models (Durrett and Levin 1994, Molofsky 1994, Pacala and Levin 1997, Bolker and Pacala 1999). A related problem is how changes in both the magnitude and the relative scale of two processes can affect population dynamics. Our present work suggests that additional information, such as direct measures of dispersal scale and direct measures of interaction type, strength, and scale may be essential to interpret patterns.

\section{ACKNOWLEDGMENTS}

Cathy Bliss and Garr Updegraff wrote the original $\mathrm{C}$ program. We thank the Department of Biology for use of its computer laboratory to run these simulations. This manuscript was improved by the comments of Alison Bennett, Michael Cain, Jean-Baptiste Ferdy, Hans De Kroon, and three anonymous reviewers. Support for this research was provided by NSF grant DEB-9527986 to J. Molofsky and NSF DEB9996221 and USDA 94-37101-0354 to J. D. Bever and J. Antonovics.

\section{Literature Cited}

Antonovics, J., and N. C. Ellstrand. 1984. Experimental studies of the evolutionary significance of sexual reproduction. I. A test of the frequency-dependent selection hypothesis. Evolution 38:103-115.

Antonovics, J., and P. Kareiva 1988. Frequency-dependent selection and competition: empirical approaches. Philo- 
sophical Transactions of the Royal Society of London Series B 319:601-613.

Augustine, D. J., and S. J. McNaughton. 1998. Ungulate effects on the functional species composition of plant communities: herbivore selectivity and plant tolerance. Journal of Wildlife Management 62:1165-1183.

Ayala, F. J. 1971. Competition between species: frequency dependence. Science 171:820-824.

Berlow, E. L., S. A. Navarrette, C. J. Briggs, M. E. Power, and B. A. Menge. 1999. Quantifying variation in the strengths of species interactions. Ecology 80:2206-2224.

Bever, J. D. 1994. Feedback between plants and their soil communities in an old field community. Ecology 75:19651977.

Bever, J. D. 1999. Dynamics within mutualism and the maintenance of diversity: inference from a model of interguild frequency dependence. Ecology Letters 2:52-61.

Bever, J. D., K. M. Westover, and J. Antonovics. 1997. Incorporating the soil community into plant population dynamics: the utility of the feedback approach. Journal of Ecology 85:561-573.

Bolker, B. M., and S. W. Pacala. 1999. Spatial moment equations for plant competition: understanding spatial strategies and the advantages of short dispersal. American Naturalist 153:575-602.

Cale, W. G., G. M. Henebry, and J. A. Yeakley. 1989. Inferring process from pattern in natural communities. BioScience 39:600-605.

Clarke, B. 1969. The evidence for apostatic selection. Heredity 24:347-352.

Connor, E. F., and D. Simberloff. 1984. Neutral models of species' co-occurrence patterns. Pages 316-331 in D. R. Strong, Jr., D. Simberloff, L. G. Abele, and A. B. Thistle, editors. Ecological communities. Princeton University Press, Princeton, New Jersey, USA.

Durrett, R., and S. Levin. 1994. The importance of being discrete (and spatial). Theoretical Population Biology 46: 363-394.

Frank, D. A., S. J. McNaughton, and B. F. Tracy. 1998. The ecology of the earth's grazing ecosystems. BioScience 48: 513-521.

Gilpin, M. E., and J. M. Diamond. 1984. Are species cooccurrences on islands non-random, and are null hypotheses useful in community ecology? Pages 297-315 in D. R. Strong, Jr., D. Simberloff, L. G. Abele, and A. B. Thistle, editors. Ecological communities. Princeton University Press, Princeton, New Jersey, USA.

Gilpin, M. E., J. M. Diamond, E. F. Connor, and D. Simberloff. 1984. Rejoinders. Pages 332-343 in D. R. Strong, Jr. D. Simberloff, L. G. Abele, and A. B. Thistle, editors Ecological communities. Princeton University Press, Princeton, New Jersey, USA.

Gleason, H. A. 1939. The individualistic concept of the plant association. American Midland Naturalist 21:92-110.

Gotelli, N. J., and G. R. Graves. 1996. Null models in ecology. Smithsonian Institution Press, Washington, D.C., USA.

Greig-Smith, P. 1983. Quantitative plant ecology. University of California Press, Berkeley, California, USA.

Greig-Smith, P., and M. J. Chadwick. 1965. Data on pattern within plant communities. III. Acacia-Capparia semi-desert scrub in the Sudan. Journal of Ecology 53:465-474.

Holley, R. A., and T. M. Liggett, 1975. Ergodic theorems for weakly interacting systems and the voter model. Annals of Probability 3:643-663.

Kareiva, P., and M. Anderson. 1988. Spatial aspects of species interactions: the wedding of models and experiments. Pages 35-50 in A. Hastings, editor. Community ecology. Lecture notes in biomathematics 77. Springer-Verlag, Berlin, Germany.

Kelley, S. E., J. Antonovics, and J. Schmitt. 1988. A test of the short-term advantage of sexual reproduction. Nature 331:714-716.

Kershaw, K. A., and J. H. H. Looney 1985. Quantitative and dynamic plant ecology. Third edition. Edward Arnold, London, UK.

Levin, D. A., and H. W. Kerster. 1974. Gene flow in seed plants. Evolutionary Biology 17:139-220.

Levin, S. A. 1992. The problem of pattern and scale in ecology. Ecology 73:1943-1967.

May, R. M., and R. M. Anderson. 1983. Epidemiology and genetics in the coevolution of parasites and hosts. Proceedings of the Royal Society of London Series B 219: 281-313.

Molofsky, J. 1994. Population dynamics and pattern formation in theoretical populations. Ecology 75:30-40.

Molofsky, J. 1999. The effect of nutrients and spacing on neighbor relations in Cardamine pensylvanica. Oikos 84: 506-514.

Molofsky, J., J. D. Bever, and J. Antonovics. 2001. Coexistence under positive frequency dependence. Proceedings of the Royal Society of London B 268:273-277.

Molofsky, J., R. Durrett, J. Dushoff, D. Griffeath, and S. Levin. 1999. Local frequency dependence and global coexistence. Theoretical Population Biology 55:270-282.

Murray, J. D. 1989. Mathematical biology. Biomathematics, volume 19. Springer Verlag, New York, New York, USA.

Pacala, S. W. 1987. Neighborhood models of plant population dynamics. 3. Models with spatial heterogeneity in the physical environment. Theoretical Population Biology 31:359392.

Pacala, S. W., and S. A. Levin. 1997. Biologically generated spatial pattern and the coexistence of competing species. Pages 204-232 in D. Tilman and P. Kareiva, editors. Spatial ecology. Princeton University Press, Princeton, New Jersey, USA.

Pacala, S. W., and J. A. Silander. 1985. Neighborhood models of plant population dynamics I. Single species models of annuals. American Naturalist 125:385-411.

Paine, R. T. 1980. Food webs, linkage interaction strength, and community infrastructure. Journal of Animal Ecology 49:667-685.

Pascual, M., and S. A. Levin. 1999. From individuals to population densities: searching for the intermediate scale of nontrivial determinism. Ecology 80:2225-2236.

Pielou, E. C. 1977. Mathematical ecology. John Wiley \& Sons, New York, New York, USA.

Price, M. V., and N. M. Waser. 1979. Pollen dispersal and optimal outcrossing in Delphinium nelsoni. Nature 277: 294-296.

Rummel, J. D., and J. Roughgarden. 1983. Some differences between invasion-structured and coevolution-structured competitive communities: a preliminary theoretical analysis. Oikos 41:477-486.

Silander, J. A., and S. W. Pacala. 1985. Neighborhood predictors of plant performance. Oecologia 66:256-263.

Smithson, A., and M. R. McNair. 1996. Frequency-dependent selection by pollinators: mechanisms and consequences with regard to behaviour of bumblebees Bombus terrestris (L.)(Hymenoptera: Apidae). Journal of Evolutionary Biology 9:571-588.

Turner, M. E., J. C. Stephens, and W. W. Anderson. 1982. Homozygosity and patch structure in plant populations as a result of nearest neighbor pollination. Proceedings of the National Academy of Sciences (USA) 79:203-207.

White, L. P. 1971. Vegetation stripes in sheet wash surfaces. Journal of Ecology 59:615-622.

Wilson, J. B., and A. D. Q. Agnew. 1992. Positive-feedback switches in plant communities. Advances in Ecological Research 23:263-335.

Wooton, J. T. 1994. Putting the pieces together: testing the independence of interactions among organisms. Ecology 75:1544-1551

Wright, S. 1943. Isolation by distance. Genetics 28:114-138.

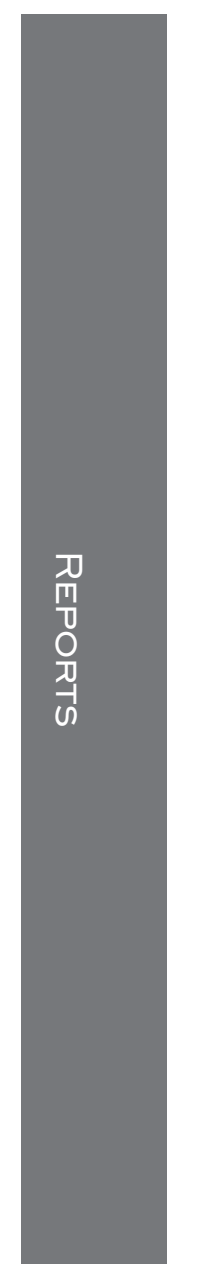

